\title{
«smarter medicine»: \\ die «Top-5-Liste» der SGC
}

Trägerschaft «smarter medicine»

Seit vielen Jahren setzt sich die Schweizerische Gesellschaft für Chirurgie (SGC) dafür ein, dass in der Weiterund Fortbildung der Chirurgen mit wissenschaftlichen Arbeiten und Messungen der Behandlungsqualität die Versorgung der Bevölkerung zu höchsten Qualitätsstandards garantiert werden kann. In der heutigen Zeit der limitierten finanziellen Mittel müssen Lösungen gefunden werden, um die Produktivität zu verbessern ohne die Qualität der Behandlung zu beinträchtigen. In dieser Hinsicht erachtet die SGC die "Choosing wisely»-Initiative und die Empfehlungen von «smarter medicine» als eine adäquate Möglichkeit, diesem Ziel näherzukommen.

Für den Bereich der Chirurgie wurde die Top-5-Liste vom American College of Surgeons in Zusammenarbeit mit verschiedenen Partnern verfasst. verfasst. Die SGC hat die amerikanischen Empfehlungen geprüft und auch für die Schweiz für vollumfänglich anwendbar und vernünftig befunden.

Die Schweizerische Gesellschaft für Chirurgie empfiehlt, folgende fünf Interventionen in der Chirurgie zu vermeiden:

1. Führen Sie bei Patienteninnen mit Brustkrebs in Stadium I oder II und klinisch negativem Lymphknotenstatus keine axilläre Dissektion ohne vorgängige Sentinel-node-Biopsie durch.

Die Sentinel-node-Biopsie hat sich für die Festlegung der Ausbreitung des Tumors bei befallenen Achsellymphknoten bewährt und führt nachgewiesenermassen zu geringeren kurz- und langfristigen Nebenwirkungen. Insbesondere sinkt dank ihr das Risiko eines Lymphödems (dauerhafte Armschwellung) deutlich.

Lassen sich bei der Untersuchung des Sentinel node keine Tumorablager feststellen, so sollten die Achsellymphknoten nicht entfernt werden.

Sind ein oder zwei Sentinel nodes noch nicht stark von Krebs befallen, wurde die Patientin brusterhaltend operiert und eine Gesamtbrustbestrahlung sowie eine dem Stadium angemessene systemische Chemotherapie geplant, so sollten die Achsellymphknoten nicht entfernt werden.

\section{Die Kampagne «smarter medicine»}

Der neu gegründeteTrägerverein verleiht der 2014 in der Schweiz lancierten Initiative "smarter medicine» Aufwind: Nebst medizinischen Fach- und Berufsorganisationen unterstützen auch $\mathrm{Pa}$ tienten- und Konsumentenorganisationen die Kampagne. Sie möchten gemeinsam die Öffentlichkeit dafür sensibilisieren, dass bei gewissen Behandlungen weniger Medizin mehr Lebensqualität für die Betroffenen bedeuten kann.

Sie knüpfen dabei an die erfolgreiche amerikanische Initiative "Choosing Wisely» an, welche zum Ziel hat, nicht nur «kluge Entscheidungen" herbeizuführen, sondern auch die offene Diskussion zwischen Ärzteschaft, den Patienten und der Öffentlichkeit zu fördern.

In den nächsten Monaten werden weitere medizinische Fachgesellschaften sogenannte Top-5-Listen mit unnützen Behandlungen in ihrem Fachbereich publizieren.

Weitere Informationen zum Trägerverein und eine Übersicht über die bestehenden Top-5-Listen unter www.smartermedicine.ch

2. Vermeiden Sie diagnostische "Ganzkörper»Computertomografien (CT) bei Patienten mit geringfügigem oder Einzeltrauma.

Ein übermässiger Einsatz von «Ganzkörper»-CTs verbessert zwar die Früherkennung von Verletzungen und kann sich gar positiv auf die Überlebensrate bei Patienten mit Mehrfachverletzungen auswirken. Dennoch gilt es bei diesen Untersuchungen auch die Strahlenexposition sowie die dadurch verbundenen Kosten zu berücksichtigen - insbesondere bei Patienten mit Traumata mit geringer Gewalteinwirkung und bei Untersuchungsbefunden, die schwere Verletzungen nicht bestätigen können.

3. Vermeiden Sie Darmkrebs-Früherkennungstests bei asymptomatischen Patienten, die eine Lebenserwartung von weniger als zehn Jahren und keine familiäre oder persönliche Vorbelastung hinsichtlich kolorektaler Neoplasie aufweisen.

Die Darmkrebsvorsorge führt nachweislich zu einer Senkung der Sterblichkeit bei dieser häufig auftretenden Erkrankung. Die Darmspiegelung ermöglicht die 


\section{Zur Ausarbeitung dieser Liste}

Das American College of Surgeons bat die Commission on Cancer, das Committee on Trauma sowie die Advisory Councils for Colon and Rectal Surgery, General Surgery and Pediatric Surgery um Empfehlungen im Zusammenhang mit der ChoosingWisely-Kampagne des American Board of Internal Medicine (ABIM). Die verschiedenen Gremien erhielten eine Beschreibung der Kampagnenziele sowie einen Link zur Website von Choosing Wisely. Darüber hinaus wurden veröffentlichte Empfehlungen von Einrichtungen, die sich bereits an der Kampagne beteiligen, in Gesprächen referenziert und geprüft. Sämtliche Empfehlungen des ACS wurden geprüft und schliesslich fünf Themen ausgewählt. Die Schweizerische Gesellschaft für Chirurgie hat die vom American College of Surgeons publizierte "Choosing Wisely"Liste geprüft und sie auch für die Schweiz als vollumfänglich anwendbar und vernünftig befunden.

Eine ausführliche Literaturliste sowie Empfehlungen von weiteren Medizinischen Fachgesellschaften sind online abrufbar unter www.smartermedicine.ch

Erkennung und Entfernung adenomatöser Polypen, einer Vorläuferläsion vieler Krebsarten. Dies verringert die Inzidenzrate der Krankheit im späteren Leben.

Übersteigen die Risiken jedoch den Nutzen, so ist diese Art des Screenings und der Überwachung ungeeignet. Die mit einer Darmspiegelung verbundenen Risiken nehmen mit dem Alter und der wachsenden Anzahl Begleiterkrankungen zu.

Das Nutzen-Risiko-Verhältnis der Darmkrebsfrüherkennung oder -überwachung sollte basierend auf früheren Vorsorgeuntersuchungen, der familiären Vorbelastung, dem voraussichtlichen Interventionsrisiko, der Lebenserwartung und der Präferenz des Patienten für jeden Fall einzeln eruiert werden.
4. Vermeiden Sie eine Eintritts- oder präoperative Röntgenuntersuchung bei ambulanten bereits untersuchten Patienten mit unauffälliger Vorgeschichte. Ein routinemässiger Röntgenthorax bei der Aufnahme oder vor der Operation ist bei ambulanten Patienten ohne spezifische Hinweise in der Vorgeschichte und/ oder in den Untersuchungsbefunden nicht angezeigt. Lediglich zwei Prozent solcher Röntgenaufnahmen führen zu einer Behandlungsänderung. Das Erstellen einer Röntgenthoraxaufnahme ist vertretbar, wenn ein Verdacht auf eine akute kardiopulmonale Erkrankung vorliegt oder im Fall von bekannten stabilen kardiopulmonaren Erkrankungen bei Patienten über 70, bei denen innerhalb der letzten sechs Monate kein Röntgenthorax erstellt wurde.

5. Führen Sie zur Abklärung einer vermuteten Blinddarmentzündung bei Kindern keine Computertomografie (CT) durch, ohne zunächst eine Ultraschalluntersuchung zu erwägen.

Obwohl eine CT zur Abklärung einer vermuteten Blinddarmentzündung bei pädiatrischen Patienten richtig ist, wird bei Kindern dennoch der Ultraschall als bildgebendes Verfahren bevorzugt. Bei nicht eindeutigen Ultraschallergebnissen kann eine CT folgen. Dieser Ansatz ist kosteneffizient, senkt mögliche Strahlenrisiken und bietet eine hervorragende Diagnosegenauigkeit. Bei erfahrenen Untersuchern liegen Sensitivität und Spezifität aktuell bei 94 Prozent. Da sich das Fachwissen unterscheiden kann, sind Strategien wie die verbesserte Diagnosekompetenz bei gemeindebasierten Ultraschallangeboten und die Entwicklung evidenzbasierter klinischer Entscheidungsregeln realistische Ziele auf dem Weg zu besseren Diagnosen ohne CTScans. 